\title{
Efficacy of multiparametric telemonitoring on respiratory outcomes in elderly people with COPD: a randomized controlled trial
}

\author{
Claudio Pedone ${ }^{1,2^{*}}$, Domenica Chiurco $^{1^{*}}$, Simone Scarlata ${ }^{1}$ and Raffaele Antonelli Incalzi ${ }^{1,3}$
}

\begin{abstract}
Background: Chronic obstructive pulmonary disease (COPD) is a highly prevalent condition associated with a high health care resource consumption and health care expenditures, driven mainly by exacerbations-related hospitalizations. Telemedicine has been proposed as a mean for timely detection of exacerbation, but the available evidence is inadequate to provide conclusive information on its efficacy. The aim of this study is to evaluate the efficacy of a telemonitoring system in reducing COPD-related hospitalizations in an elderly population with COPD.

Methods: This is a parallel arms, randomized trial including patients aged 65 or older with COPD in GOLD stages II and III enrolled in a Pulmonary Medicine outpatient facility. Patients were randomly assigned to receive a non-invasive system able to telemonitor vital signs (oxygen saturation, heart rate, near-body temperature, overall physical activity) or standard care, and were followed up for 9 months. The outcome measures were the number of exacerbations and exacerbation-related hospitalization.

Results: Fifty patients were included in the telemonitoring group and 49 in the control group. The incidence rate of respiratory events was 28/100 person/years in the telemonitoring group vs. $42 / 100$ person/years in the control group (incidence rate ratio: $0.67,95 \% \mathrm{Cl}: 0.32-1.36$ ). The corresponding figures for hospital admissions where 13/100 person/years and 20/100 person/years, respectively (IRR: 0.66, 95\% Cl: $0.21-1.86)$.

Conclusions: In our study, COPD patients followed up with the aid of a multiparametric remote monitoring system experienced a lower rate of exacerbations and COPD-related hospitalizations compared to patients followed up using the standard model of care. These results need to be replicated in larger studies before they can be applied to the general COPD population. Trial registration number: NCT01481506 (clinicaltrials.gov). Funding: co-financed by Lazio Region and Intersistemi Inc.
\end{abstract}

Keywords: Chronic obstructive pulmonary disease, Aged, Telemonitoring

\section{Background}

Chronic obstructive pulmonary disease (COPD) is a highly prevalent condition that is expected to be the third cause of death worldwide by 2020 [1]. It is also associated with important risk of disability with a related very high use of health care resources. It has been reported that hospital care, which is mainly driven by exacerbations, is the main component of health care expenditures, and exacerbations prevention can reduce costs [2]. Comorbidity,

\footnotetext{
*Correspondence: c.pedone@unicampus.it; d.chiurco@unicampus.it

${ }_{1}^{1}$ Area di Geriatria. Università Campus Biomedico, Roma, Italy

2Fondazione "A. Sordi", Roma, Italy

Full list of author information is available at the end of the article
}

mainly cardiovascular, substantially contributes to health care costs [3]. Selected interventions, such as patient's education and dedicated health programs [4] as well as some pharmacological measures [5], have achieved this objective. Telemedicine has also been tried as a mean for detecting worsening health status and to implement timely interventions [6]. The relevant experience, however, is scarce because the vast majority of the studies are small and lack a control group [7].

Telemonitoring of COPD patients has been so far based upon programmed or on demand vocal interaction or periodical transmission of expiratory peak flow or transcutaneous oxygen saturation $\left(\mathrm{SaO}_{2}\right)$ [8-11]. All 
these interventions are monodimensional in nature and episodic or periodic in their timing, and have yielded mixed results with respect to cost reduction and quality of life improvement. COPD, mainly in the presence of cardiovascular comorbidity, is multidimensional in its impact on health status $[12,13]$.

Furthermore, older age can modulate the clinical expression of COPD and its exacerbations by increasing the relative weight of non respiratory symptoms such as muscle weakness or dizziness [14]. Thus, a multidimensional approach seems desirable for a comprehensive monitoring of COPD patients, mainly if in the geriatric age, to identify timely changes in health dimensions heralding COPD exacerbations. Such a monitoring system has to be accurate, but also simple, economic and user-friendly in order to be useful in clinical practice.

In the present study we present a randomized, parallelgroup trial of a multiparametric remote monitoring system measuring physical activity, cardiac and respiratory rates, near-body temperature and peripheral oxygen saturation.

\section{Methods}

\section{Study design}

This was a single-center, unmasked, randomized trial with 9 months of follow-up of multi-parametric telemonitoring vs. standard care. Patients were recruited among those attending the Pneumology outpatient facilities of the Campus Biomedico University in Rome by a study researcher. Eligibility criteria were age $\geq 65$ years and diagnosis of COPD stage II or III according to the GOLD criteria (http://www.goldcopd.org): forced expiratory volume in the first second (FEV1) / forced vital capacity $(\mathrm{FVC})<0.7$ and $30 \%<\mathrm{FEV} 1$ percent predicted $<80 \%$. We excluded patients with a life expectancy $<6$ months and those with cognitive impairment severe enough to preclude the use of the telemonitoring device. The study protocol was approved by the Ethical Committee at the Campus Biomedico University (\#29/2009).

\section{Intervention}

The "SweetAge" monitoring system was developed specifically for this study by a collaborative group of including both research centers and business enterprises (see "Acknowledgements"). It is made up by three main components on the patient's side:

1. a wristband that contained the sensors for heart rate, physical activity, near-body temperature, and galvanic skin response. The wristband also contained a bluetooth transmitter;

2. a commercial pulse-oxymeter (Nonin Medical Inc.) connected to a bluetooth transmitter coupled with the wristband;
3. a commercial cellular telephone coupled with the wristband via a bluetooth connection. The telephone was equipped with a software that allowed the reception of the data transmitted by the wristband and acted as a gateway to send the data to the monitoring system.

The system was extensively tested before the beginning of the trial on both healthy controls and COPD patients. The information obtained were compared to those objectively measured, and a satisfactory agreement was obtained. It should be noted that for oxygen saturation the "SweetAge" system used a commercial device, and therefore for this parameter we only tested the dependability of the transmission system.

A dedicated software allowed real-time monitoring of the information gathered by the devices listed above. This monitor system was web-based and accessed through a secure connection using a standard Internet browser. The operation of the components of the patient's side were mostly automatic: the patients only had to wear the wristband and to turn it on, while keeping the cell phone in the range of the bluetooth transmission. The patient was not aware of wristband operations, except for oxygen saturation: a sound reminded the patient to wear the pulse-oxymeter when the measurement were scheduled.

The system was set up to perform 5 measurement of each parameter every three hours. Oxygen saturation was measured over 1 minute, for the others parameters five measurements of 1 minute each were performed at a sampling rate of $60 \mathrm{~Hz}$. The measurements were averaged by the monitor system and displayed as the mean value to the operator. The individual values were available on demand. The patient could access the data at the moment of measurement, as they were displayed on the telephone's screen.

The data received were evaluated every day by a physician skilled in the care of respiratory patients. The monitor system displayed an alert when a measurement was outside the predefined range. The limits for these alerts could be customized for the individual patient by the user of the system on the basis of the patient's specific clinical situation. This system, however, was intended for monitoring only, and the patients were instructed to contact their usual health care provider in case of need.

In case of abnormal readings, the physician contacted the patient to verify whether their symptoms had worsened or new symptoms had arisen. In this event, the patient's adherence to therapy was checked and, if unsatisfactory, individually tailored interventions promoting adherence were carried up. Otherwise, a diagnosis of exacerbation was made and, on the basis of its severity a office appointment (for mild exacerbations) or a hospital admission was scheduled. 


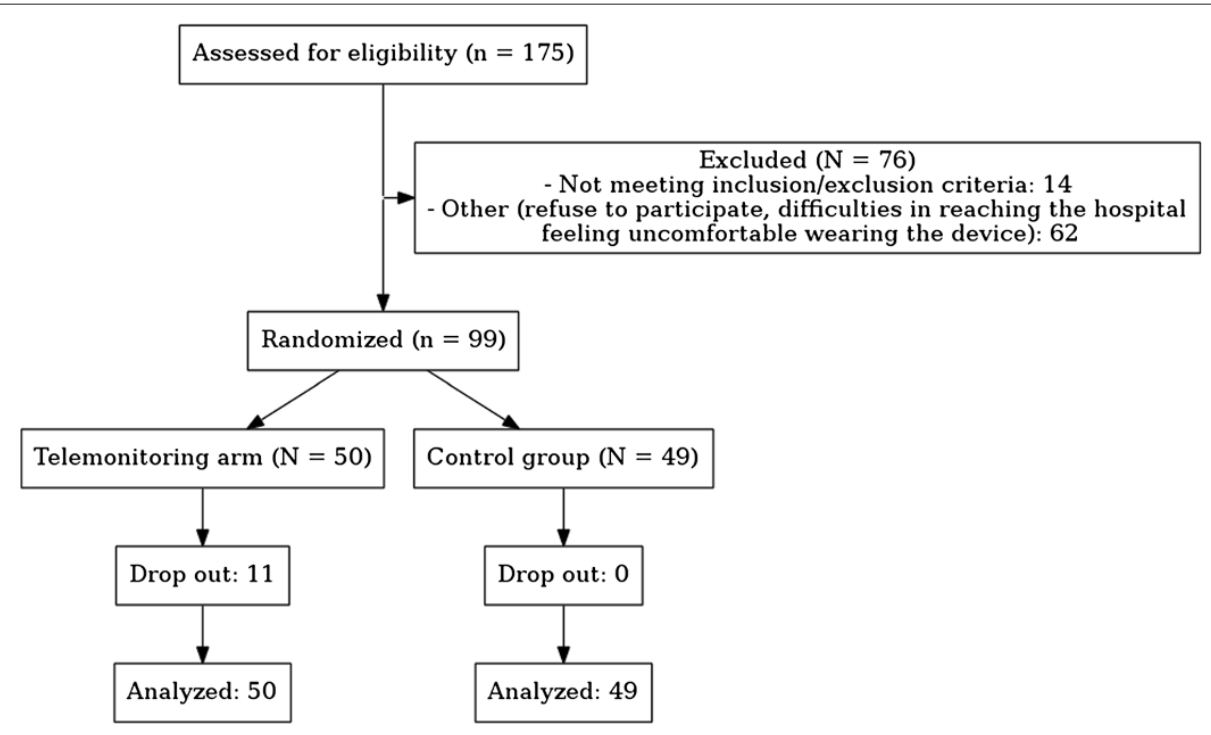

Figure 1 Flow diagram of the study.

\section{Outcome}

The outcome measures of these study were: number of exacerbations, defined as a sustained worsening of the patient's condition, from the stable state and beyond normal day-to-day variations, that is acute in onset and necessitates a change in regular medications [15], and number of hospital admissions. All the information were gathered from clinical records, when available, or in person/telephone interview.

\section{Sample size}

We anticipated a mean of 1.5 exacerbation/patient/year [16], corresponding to a risk of $81 \%$ of having the event. Allowing for a type I error rate of $5 \%$, we calculated that a sample size of 50 patients per group would provide a $80 \%$ power of detecting a $30 \%$ reduction of the risk. Participants were allocated to the study groups in a 1:1 ratio using a computer-generated random list of number.

\section{Analytic approach}

The demographic and functional characteristics of the two groups were compared using descriptive statistics and t-test or chi-square test, as appropriate. Physical disability at baseline was evaluated using the basic Activities of Daily Living [17] that investigates 6 domains providing a score ranging from 0 (total disability) to 6 (no disability). Cognitive function was investigated using the MiniMental State Examination [18], a scale providing a score ranging from 0 to 30 , with lower scores $(<24)$ indicating possible cognitive impairment. The risk for the outcome of interest was evaluated by calculating incidence rates and incidence rate ratios along with $95 \%$ confidence intervals. All the analyses were performed using the "intention to treat" approach. The analyses were performed using $\mathrm{R}$ Statistical Software version 2.14 for Linux (R Foundation for Statistical Computing, Wien, Austria, 2011).

\section{Results and discussion}

The flow diagram of the study is reported in Figure 1. Overall we followed up 99 patients with a mean age of 74 years. Over the follow-up period, $20 \%$ of patients dropped out from the study, but were followed for the outcomes of interest. Reasons for drop out were: patient feeling uncomfortable with the wearable device or thinking that they disrupt the daily life rhythm.

About two-thirds (68\%) of participants were men. The average FEV1 was $54 \%$ of predicted (range $23 \%-77 \%$ ). As shown in Table 1, there were no differences in the main characteristics of participants in the two groups, although

Table 1 Characteristics of participants

\begin{tabular}{lccc}
\hline & SweetAge $(\mathbf{N}=\mathbf{5 0})$ & Controls $(\mathbf{N}=\mathbf{4 9})$ & $\mathbf{P}$ \\
\hline Age & $74.1(6.4)$ & $75.4(6.7)$ & 0.340 \\
Gender (Men) & $72 \%$ & $63 \%$ & 0.475 \\
FEV1 \% & $52.5(14.9)$ & $55.4(15.8)$ & 0.413 \\
FVC \% & $78.8(16.5)$ & $78.5(16.9)$ & 0.947 \\
GOLD stages & - & - & \\
I & $57.1 \%$ & $59.5 \%$ & 0.984 \\
II & $42.9 \%$ & $40.5 \%$ & \\
III & - & - & \\
IV & $5.6(1.0)$ & $5.1(1.3)$ & 0.157 \\
Mean ADL score & $28.1(2.1)$ & $26.7(4.0)$ & 0.084 \\
MMSE score & & & \\
\hline
\end{tabular}




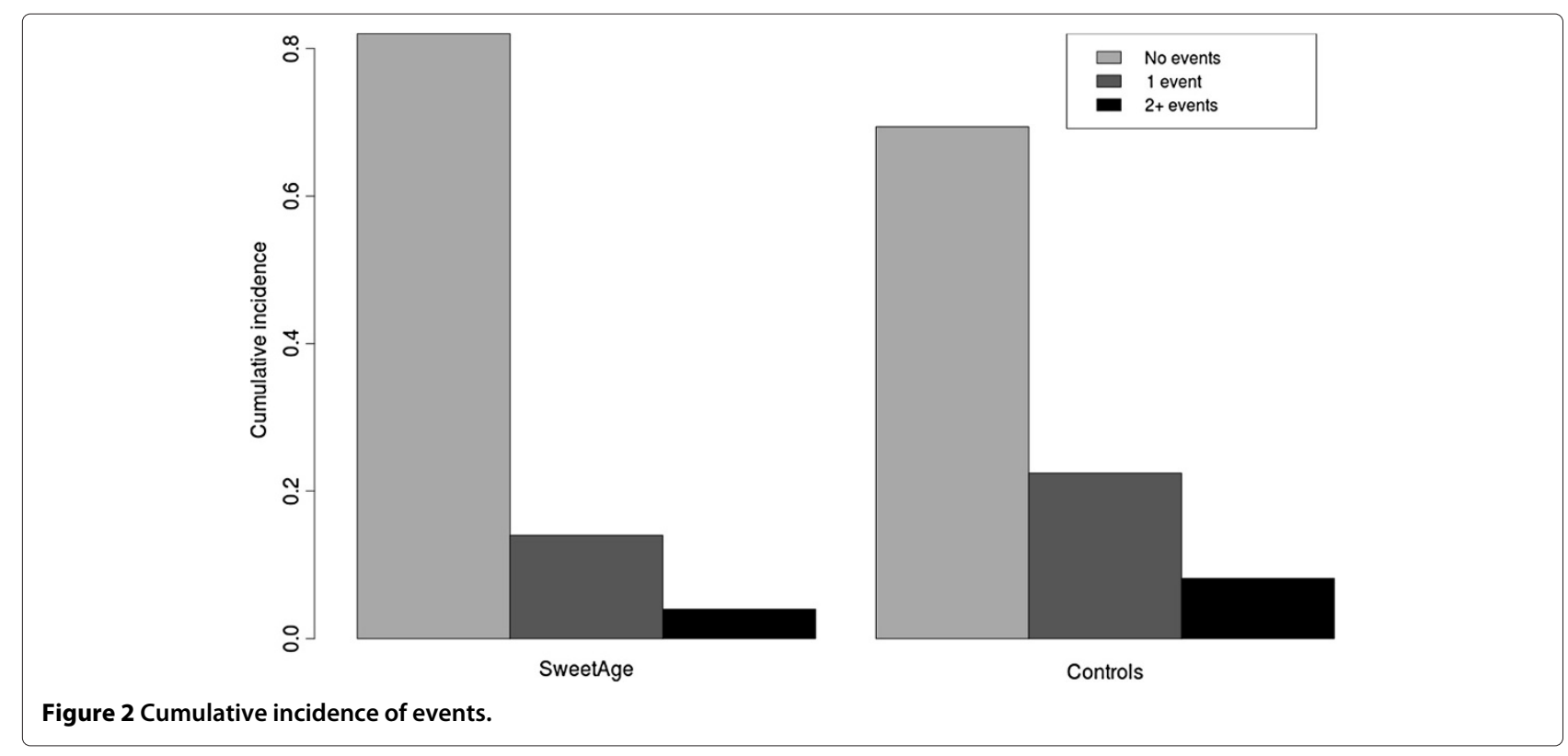

controls had a slighly worse performance in instrumental activities of daily living.

On average, the SweetAge equipment sent data for 150 days/patient, with a mean of 4 measurements/day. Considering the days in which the system was actually used, the proportion of days with at least 3 measurements ranged from $0 \%$ to $97 \%$ (mean: $60 \%$, interquartile range: $50 \%-80 \%)$. Per protocol, all the events detected by the telemonitoring system triggered at least a telephone call by the study researcher. Only one out of fourteen such calls revealed a self-limiting problem, while in all other cases other actions were undertaken.

Overall, we observed 19 events in the control group and 13 events in the experimental group. Among controls, $15 / 49$ (31\%) participants experienced at least 1 respiratory event, compared to $9 / 50(18 \%)$ in the SweetAge group. The incidence of multiple events was $8 \%$ among controls and $4 \%$ in the SweetAge group (Figure 2). In terms of incidence rate, we observed 28 events $/ 100$ person year in the SweetAge group and 42 events/100 person year in the control group, with and incidence rate ratio of 0.67 (95\% CI: 0.32 - 1.36) (Table 2).

In both groups, about half of the events have been hospital admissions (46\% and $47 \%$ in the SweetAge and control

Table 2 Respiratory events during follow-up

\begin{tabular}{lcc}
\hline & SweetAge $(\mathbf{N}=\mathbf{5 0})$ & Controls $(\mathbf{N}=\mathbf{4 9})$ \\
\hline $\begin{array}{l}\text { Cumulative incidence } \\
\text { of events }\end{array}$ & $18 \%$ & $31 \%$ \\
$\begin{array}{l}\text { Cumulative incidence } \\
\text { of multiple events }\end{array}$ & $4 \%$ & $8 \%$ \\
Incidence rate & & \\
Incidence rate ratio & $28 / 100$ person-year & $42 / 100$ person-year \\
\end{tabular}

group, respectively). The corresponding incidence rate was $13 / 100$ person year in the SweetAge group and 20/100 person year in the control group, with an incidence rate ratio of 0.66 (95\% CI: 0.21 - 1.86). The average length of stay was 9.7 days in the SweetAge group and 6.9 days in the control group.

The SweetAge system revealed a worsening of peripheral oxygen saturation in the days preceding most of the respiratory events (see Figure 3), allowing a timely intervention (e.g. drug regimen modifications) or, in most severe cases, to plan the hospital admission avoiding an emergency room visit.

In our study, COPD patients followed up with the aid of a multiparametric remote monitoring system experienced a lower rate of exacerbations and COPD-related hospitalizations compared to patients followed up using the standard model of care. The presence of a control group and the common operational framework guarantees for the reliability of our data. In addition, we showed that respiratory events can actually be timely detected by a wearable system whose operations are fully automated and require very little skills on the patient's side.

Previous studies provided controversial results on the possibility of preventing exacerbations through a telehealth approach. A recent review, however, pointed out that only four out of a thousand studies taken into account had a control group [19]. A meta-analysis on this topic [20] concluded that while home telemonitoring and telephone support seem to be effective in reducing the rate of hospitalization and emergency room visits, the evidence on number of hospital days and on mortality are conflicting. In our sample, telemonitoring could cut by $33 \%$ the risk for hospitalizations. However, the average length of stay was longer in the study than in the control 


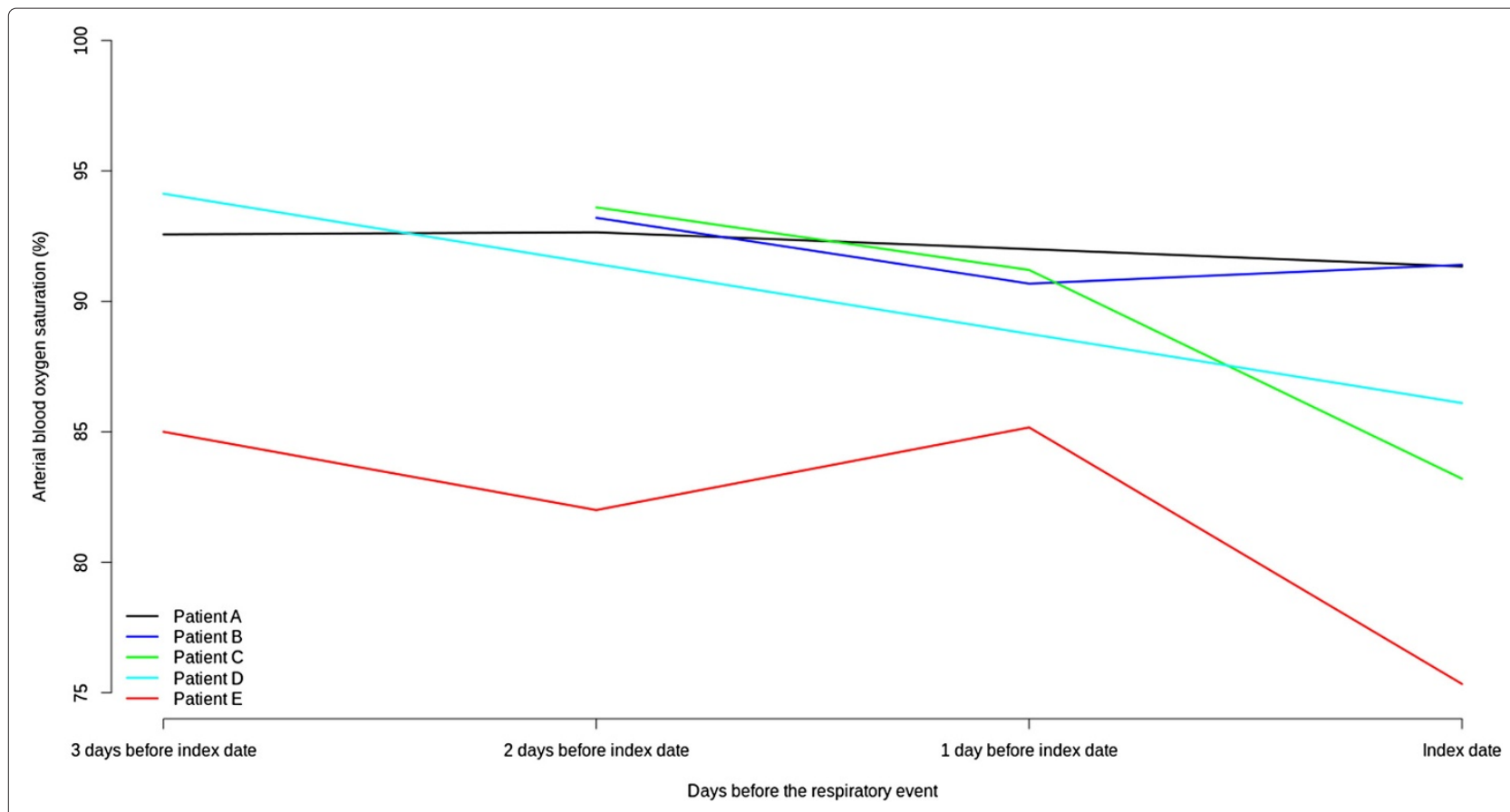

Figure 3 Arterial oxygen saturation in the three days preceding the respiratory event. Trend of the arterial oxygen saturation in the three days before a respiratory event. Each line represents data from a single patient.

group. This finding likely is consistent with a lower threshold for hospitalization in the control group, i. e. for a more stringent selection of patients needing hospitalization in the study group. Thus, telemonitoring may be effective promoting home care of less severe COPD exacerbations. This result is in keeping with data showing that a "hospital at home" approach is safe and successful in selected patients [21].

Telephone contacts had a second step role in our telemonitoring design. Indeed, despite some positive evidences [6,22,23], a recent metanalysis of 11 studies shows that telephone based care was associated with greater mortality than usual care [20] in people with COPD. Furthermore, this approach could not benefit patients with heart failure, a condition that, like COPD, is dominated by dyspnea, even in a very large and well conducted trial [24]. It should be noted that dyspnea, the symptom most commonly collected in the telephone interview of cardiac or respiratory patients, is subject to large interindividual variability: non respiratory symptoms such as asthenia, leg discomfort/edema dominate the clinical proportion in a consistent proportion of COPD patients experiencing severe, even life-threatening exacerbations [14]. Furthermore, in patients able to perform physical activity, the ergoreflex, which originates from overworking respiratory muscles, causes peripheral sympathetic overdischarge and, eventually, peripheral vasoconstriction, muscle ischemia and fatigue [25]. As a consequence, patients decrease their already limited activity, which prevents the worsening of dyspnoea and postpones the recognition of the exacerbation. On these bases, we resolved to monitor also non respiratory parameters and to consider the telephonic interview as a second step intervention driven by changes in monitored parameters.

Among the parameters monitored, only oxygen saturation could timely identify COPD exacerbations. This reflects the expected high specificity of $\mathrm{O}_{2}$ desaturation: it is very unlikely that a condition other than respiratory exacerbation accounts for this event. However, minor differences in the precritical multiparametric profiles were evident. The relatively short duration of the study and, thus, the limited number of critical events prevented us from verifying whether well defined and repeatable patterns can be identified. A larger study might clarify this issue, which is of practical as well conceptual interest. Indeed, defining different trajectories towards the exacerbation would also shed light on phenotypic variability of COPD.

This study has some limitations. We had a lower incidence of events than expected and consequently the confidence interval around our point estimates were large and we cannot exclude that the better outcomes observed in the telemonitoring group are due to random error. The low rate of events observed may have different causes, including the improved management of COPD in the latest years. Evidence in support of a more aggressive 
treatment of both COPD and their comorbidities, leading to hospitalization only of more severe exacerbations has been reported by different studies [26,27]. At any rate, despite the reduced statistical power, our results are in line with other showing that remote arterial oxygen saturation monitoring can reduce hospitalizations in people with very severe COPD [28]. Larger studies, however, are needed to confirm the effectiveness of this telemonitoring system. Another potential limitation is that cannot exclude that results were partly due to the education provided to the patients, although cases and controls were given the same basic instructions with regard to perception of changing health status and were offered the same ambulatory support. However, cases more frequently attended the ambulatory to have information on the use of the device, and this might have been a source of difference between groups.

This study also has some methodological and technological strengths. On the methodological side, it was a rigorously designed and conducted randomized trial, which guarantees for reliability of results. On the technological side, at variance form the traditional telemonitoring of COPD patients, monodimensional in nature or, if multidimensional, based on cumbersome technological tools and procedures [20], our telemonitoring system was very easy to use: the patient had to do little more than wearing the device. Moreover, it allowed to monitor the patients at different times of the day and in different conditions (e.g. indoor vs. outdoor, at rest and during exercise, etc.), and was suitable for both chronic monitoring and "on demand" use.

\section{Conclusions}

In conclusion, our data suggest that, compared to standard care, a multiparametric remote monitoring system may reduce COPD exacerbations rates and COPD-related hospitalizations. This favorable experience needs to be replicated in larger populations in order to achieve sufficient statistical power and to derive algorithms able to identify the exacerbation in its "subclinical" stage according to different individual profiles.

Unfortunately, enormous economic efforts are commonly devoted to pharmacologic, but not to technological innovation. This trial adds further evidence to the concept that telemonitoring is worth of implementation and diffusion as a component of the care of COPD.

\footnotetext{
Abbreviations

COPD: Chronic Obstructive Pulmonary Disease; GOLD: Global initiative for Obstructive Lung Disease; $\mathrm{FEV}_{1}$ : Forced Expiratory Volume in the first second; FVC: Forced Vital Capacity; $\mathrm{SaO}_{2}$ : Arterial oxygen saturation.
}

\section{Competing interests}

None of the authors has any conflict of interest to disclose in relation to this manuscript. In particular, none of the authors has financial relationship of any kind with Intersistemi SpA or Evolvo srl.

\section{Authors' contributions}

CP participated in designing the monitoring system and the study. He drafted the manuscript in collaboration with RAl and performed the statiscal analyses. DC participated in recruiting the participants and in the monitoring activity.

She revised the manuscript for important intellectual content. SS participated in the follow-up of the control group. He has revised the manuscript for important intellectual content. RAl participated in designing the study. He drafted the manuscript in collaboration with CP and revised it for important intellectual content. All authors read and approved the final manuscript.

\section{Acknowledgements}

The SweetAge project has been funded by the Lazio Region through the "Finanziaria Lazio per lo Sviluppo (FILAS)". The SweetAge telemonitoring system is the result a cooperative effort that included business enterprises (Intersistemi SpA, Rome, and Evolvo srl, Rome) and research centers (Campus Biomedico University, Rome, and the CATTID at the University of Rome "La Sapienza"). The project was carried out under the management of Go Management Consulting, Rome. The clinical data were independently collected by researchers at Campus Biomedico University, the industrial partners had no access to the clinical data and did not participate in any way in the preparation of this report.

\section{Author details}

${ }^{1}$ Area di Geriatria. Università Campus Biomedico, Roma, Italy. ${ }^{2}$ Fondazione "A Sordi", Roma, Italy. ${ }^{3}$ Fondazione "S Raffaele - Cittadella della Carità", Taranto, Italy.

Received: 25 June 2012 Accepted: 10 February 2013

Published: 6 March 2013

\section{References}

1. Murray CJ, Lopez AD: Alternative projections of mortality and disability by cause 1990-2020: Global Burden of Disease Study. Lancet 1997, 349(9064):1498-1504. [http://dx.doi.org/10.1016/S01406736(96)07492-2]

2. Dalal AA, Christensen L, Liu F, Riedel AA: Direct costs of chronic obstructive pulmonary disease among managed care patients. Int $\mathrm{J}$ Chron Obstruct Pulmon Dis 2010, 5:341-349. [http://www.ncbi.nlm.nih. gov/pubmed/21037958] [PMID: 21037958]

3. Simon-Tuval T, Scharf SM, Maimon N, Bernhard-Scharf BJ, Reuveni H, Tarasiuk A: Determinants of elevated healthcare utilization in patients with COPD. Respir Res 2011, 12:7. [http://www.ncbi.nlm.nih. gov/pubmed/21232087] [PMID: 21232087]

4. Steuten LMG, Lemmens KMM, Nieboer AP, Vrijhoef HJM: Identifying potentially cost effective chronic care programs for people with COPD. Int J Chron Obstruct Pulmon Dis 2009, 4:87-100. [http://www.ncbi. nlm.nih.gov/pubmed/19436687] [PMID: 19436687].

5. Seemungal TAR, Hurst JR, Wedzicha JA: Exacerbation rate, health status and mortality in COPD-a review of potential interventions. Int J Chron Obstruct Pulmon Dis 2009, 4:203-223. [http://www.ncbi.nlm. nih.gov/pubmed/19554195] [PMID: 19554195].

6. Casas A, Troosters T, Garcia-Aymerich J, Roca J, Hernández C, Alonso A del Pozo F, de Toledo P, Antó JM, Rodríguez-Roisín R, Decramer M Integrated care prevents hospitalisations for exacerbations in COPD patients. Eur Respir J 2006, 28:123-130. [http://www.ncbi.nlm.nih.gov/ pubmed/16611656] [PMID: 16611656].

7. McKinstry B, Pinnock H, Sheikh A: Telemedicine for management of patients with COPD? Lancet 2009, 374(9691):672-673. [http://www. ncbi.nlm.nih.gov/pubmed/19716948] [PMID: 19716948]

8. de Toledo P, Jiménez S, del Pozo F, Roca J, Alonso A, Hernandez C: Telemedicine experience for chronic care in COPD. IEEE Trans Inf Technol Biomed 2006, 10(3):567-573. [http://www.ncbi.nlm.nih.gov/ pubmed/16871726] [PMID: 16871726]

9. Lewis KE, Annandale JA, Warm DL, Hurlin C, Lewis MJ, Lewis L: Home telemonitoring and quality of life in stable, optimised chronic obstructive pulmonary disease. J Telemed Telecare 2010, 16(5):253-259. [http://www.ncbi.nlm.nih.gov/pubmed/20483881] [PMID: 20483881].

10. Paré G, Sicotte C, St-Jules D, Gauthier R: Cost-minimization analysis of a telehomecare program for patients with chronic obstructive pulmonary disease. Telemed J E Health 2006, 12(2):114-121. [http:// www.ncbi.nlm.nih.gov/pubmed/16620165] [PMID: 16620165]. 
11. Trappenburg JCA, Niesink A, de Weert-van Oene GH, van der Zeijden $H$, van Snippenburg R, Peters A, Lammers JJ, Schrijvers AJP: Effects of telemonitoring in patients with chronic obstructive pulmonary disease. Telemed J E Health 2008, 14(2):138-146. [http://www.ncbi.nlm. nih.gov/pubmed/18361703] [PMID: 18361703].

12. Celli BR: Predictors of mortality in COPD. Respir Med 2010 104(6)::773-779. [http://www.ncbi.nlm.nih.gov/pubmed/20417082] [PMID: 20417082].

13. Sin DD, Anthonisen NR, Soriano JB, Agusti AG: Mortality in COPD: Role of comorbidities. Eur Respir J 2006, 28(6):1245-1257. [http://www.ncbi. nlm.nih.gov/pubmed/17138679] [PMID: 17138679].

14. Incalzi RA, Fuso L, Serra M, Basso S, Carosella L, Tramaglino LM, Pistelli R, Carbonin P: Exacerbated chronic obstructive pulmonary disease: a frequently unrecognized condition. $J$ Intern Med 2002, 252:48-55. [http://www.ncbi.nlm.nih.gov/pubmed/12074738] [PMID: 12074738].

15. Rodriguez-Roisin R: Toward a consensus definition for COPD exacerbations. Chest 2000, 117(5 Suppl 2):398S-401S. [http://www.ncbi. nlm.nih.gov/pubmed/10843984] [PMID: 10843984].

16. Donaldson GC, Seemungal TAR, Patel IS, Lloyd-Owen SJ, Wilkinson TMA Wedzicha JA: Longitudinal changes in the nature, severity and frequency of COPD exacerbations. Eur Respir J 2003, 22(6):931-936.

17. Katz S, Ford AB, Moskowitz RW, Jackson BA, Jaffe MW: Studies of illness in the aged. The index of ADL: A standardized measure of biological and psychosocial function. JAMA 1963, 185:914-919.

18. Folstein MF, Robins LN, Helzer JE: The mini-mental state examination. Arch Gen Psychiatry 1983, 40(7):812.

19. Smith SM, Brame A, Kulinskaya E, Elkin SL: Telemonitoring and intermediate care. Chest 2011, 139(3):731-732. [http://www.ncbi.nlm. nih.gov/pubmed/21362670] [PMID: 21362670].

20. Polisena J, Tran K, Cimon K, Hutton B, McGill S, Palmer K, Scott RE: Home telehealth for chronic obstructive pulmonary disease: a systematic review and meta-analysis. J Telemed Telecare 2010, 16(3):120-127. [http://www.ncbi.nlm.nih.gov/pubmed/20197355] [PMID: 20197355].

21. Ram FSF, Wedzicha JA, Wright J, Greenstone M: Hospital at home for acute exacerbations of chronic obstructive pulmonary disease. Cochrane Database Syst Rev 2003, 4:CD003573. [http://www.ncbi.nlm.nih. gov/pubmed/14583984] [PMID: 14583984].

22. Wennberg DE, Marr A, Lang L, O'Malley S, Bennett G: A randomized trial of a telephone care-management strategy. N Engl J Med 2010, 363(13):1245-1255. [http://www.ncbi.nlm.nih.gov/pubmed/20860506] [PMID: 20860506].

23. Bourbeau J, Julien M, Maltais F, Rouleau M, Beaupré A, Bégin R, Renzi P, Nault D, Borycki E, Schwartzman K, Singh R, Collet J: Reduction of hospital utilization in patients with chronic obstructive pulmonary disease: a disease-specific self-management intervention. Arch Intern Med 2003, 163(5):585-591. [http://www.ncbi.nlm.nih.gov/pubmed/ 12622605] [PMID: 12622605].

24. Chaudhry SI, Mattera JA, Curtis JP, Spertus JA, Herrin J, Lin Z, Phillips CO, Hodshon BV, Cooper LS, Krumholz HM: Telemonitoring in patients with heart failure. N Eng/ J Med 2010, 363(24):2301-2309. [http://www.ncbi. nlm.nih.gov/pubmed/21080835] [PMID: 21080835].

25. Schmidt H, Francis DP, Rauchhaus M, Werdan K, Piepoli MF: Chemo- and ergoreflexes in health, disease and ageing. Int J Cardiol 2005 98(3):369-378. [http://www.ncbi.nlm.nih.gov/pubmed/15708167] [PMID: 15708167].

26. Fremault A, Janssens W, Beaucage F, Celis G, Pérez-Bogerd S, Decramer M: Modification of COPD presentation during the last 25 years. COPD 2010, 7(5):345-351. [http://dx.doi.org/10.3109/15412555.2010.510546].

27. Almagro P, Salvadó M, Garcia-Vidal C, Rodriguez-Carballeira M, Delgado $M$, Barreiro $B$, Heredia JL, Soriano JB: Recent improvement in long-term survival after a COPD hospitalisation. Thorax 2010, 65(4):298-302. [http://dx.doi.org/10.1136/thx.2009.124818].

28. Vitacca M, Bianchi L, Guerra A, Fracchia C, Spanevello A, Balbi B, Scalvini S: Tele-assistance in chronic respiratory failure patients: a randomised clinical trial. Eur Respir J 2009, 33(2):411-418. [http://www.ncbi.nlm.nih. gov/pubmed/18799512] [PMID: 18799512].

doi:10.1186/1472-6963-13-82

Cite this article as: Pedone et al: Efficacy of multiparametric telemonitoring on respiratory outcomes in elderly people with COPD: a randomized controlled trial. BMC Health Services Research 2013 13:82.

\section{Submit your next manuscript to BioMed Central and take full advantage of:}

- Convenient online submission

- Thorough peer review

- No space constraints or color figure charges

- Immediate publication on acceptance

- Inclusion in PubMed, CAS, Scopus and Google Scholar

- Research which is freely available for redistribution

Submit your manuscript at www.biomedcentral.com/submit 